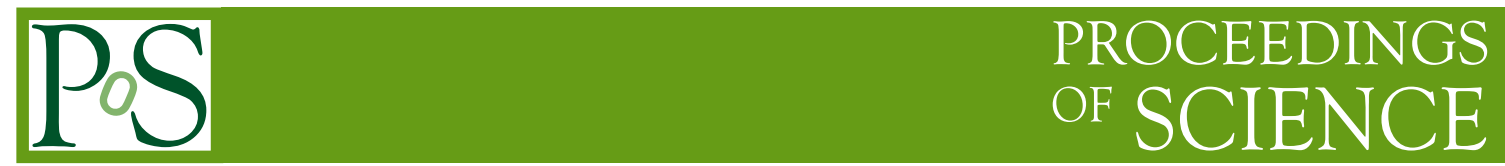

\title{
Evolution of gluon TMDs from low to moderate $\mathbf{x}$
}

\section{Ian Balitsky ${ }^{* \dagger}$}

JLab, 12000 Jefferson Ave, Newport News, VA 23606, USA,

and

Phys. Dept., Old Dominion Univ., 4600 Elkhorn Ave, Norfolk, VA 23529, USA

E-mail: balitsky@jlab.org

I discuss how the rapidity evolution of gluon transverse momentum dependent distribution changes from nonlinear evolution at small $x \ll 1$ to linear evolution at moderate $x \sim 1$.

QCD Evolution 2017

22-26 May, 2017

Jefferson Lab Newport News, VA - USA

* Speaker.

${ }^{\dagger}$ A footnote may follow. 


\section{Introduction}

The TMDs $[1,2,3]$ (also called unintegrated parton distributions) are widely used in the analysis various scattering processes in JLab and elsewhere on low-energy accelerators. However, since at the future EIC accelerator the majority of the produced particles will be gluons (turning to hadrons due to confinement) one needs to study also the evolution of gluon TMDs. The TMD generalizes the usual concept of parton density by allowing PDFs to depend on intrinsic transverse momenta in addition to the usual longitudinal momentum fraction variable. The unintegrated gluon distribution is defined as [4]

$$
\begin{aligned}
& \mathscr{D}\left(x_{B}, k_{\perp}, \eta\right)=\int d^{2} z_{\perp} e^{i(k, z)_{\perp}} \mathscr{D}\left(x_{B}, z_{\perp}, \eta\right), \\
& \alpha_{S} \mathscr{D}\left(x_{B}, z_{\perp}, \eta\right)=\frac{-x_{B}^{-1}}{8 \pi^{2}(p \cdot n)} \int d u e^{-i x_{B} u(p n)}\left\langle p\left|\mathscr{F}_{\xi}^{a}\left(z_{\perp}+u n\right)\left[z_{\perp}+\infty n, \infty n\right]^{a b} \mathscr{F}^{b \xi}(0)\right| p\right\rangle
\end{aligned}
$$

where $|p\rangle$ is an unpolarized target with momentum $p$ (typically proton) and $\infty$ can be plus or minus infinity. Hereafter we use the notation

$$
\mathscr{F}_{\xi}^{a}\left(z_{\perp}+u n\right) \equiv\left[\infty n+z_{\perp}, u n+z_{\perp}\right]^{a m} n^{\mu} g F_{\mu \xi}^{m}\left(u n+z_{\perp}\right)
$$

where $[x, y]$ denotes straight-line gauge link connecting points $x$ and $y$ :

$$
[x, y] \equiv \mathrm{P} e^{i g \int d u(x-y)^{\mu} A_{\mu}(u x+(1-u) y)}
$$

There are more involved definitions with Eq. (1.1) multiplied by some Wilson-line factors [3, 5] following from CSS factorization [6] but we will discuss the "primordial" TMD (1.1).

\section{Rapidity factorization and evolution}

In the spirit of high-energy OPE, the rapidity of the gluons in the operator (1.2) is restricted from above by the "rapidity divide" $\eta$ separating the impact factor and the matrix element

$$
A_{\mu}^{\eta}(x)=\int \frac{d^{4} k}{16 \pi^{4}} \theta\left(e^{\eta}-|\alpha|\right) e^{-i k \cdot x} A_{\mu}(k)
$$

where the Sudakov variable $\alpha$ is defined as usual, $k=\alpha p_{1}+\beta p_{2}+k_{\perp}$. We define the light-like vectors $p_{1}$ and $p_{2}$ such that $p_{1}=n$ and $p_{2}=p-\frac{m^{2}}{s} n$, where $p$ is the momentum of the target particle of mass $m$. We use metric $g^{\mu v}=(1,-1,-1,-1)$ so $p \cdot q=\left(\alpha_{p} \beta_{q}+\alpha_{q} \beta_{p}\right) \frac{s}{2}-(p, q)_{\perp}$. For the coordinates we use the notations $x_{\bullet} \equiv x_{\mu} p_{1}^{\mu}$ and $x_{*} \equiv x_{\mu} p_{2}^{\mu}$ related to the light-cone coordinates by $x_{*}=\sqrt{\frac{s}{2}} x_{+}$and $x_{\bullet}=\sqrt{\frac{s}{2}} x_{-}$. Also, hereafter we use the notation $\left[\infty, z_{*}\right]_{z} \equiv\left[\infty_{*} p_{1}+z_{\perp}, \frac{2}{s} z_{*} p_{1}+z_{\perp}\right]$ where $[x, y]$ stands for the straight-line gauge link connecting points $x$ and $y$ as defined in Eq. (1.3). Our convention is that the Latin Lorentz indices always correspond to transverse coordinates while Greek Lorentz indices are four-dimensional.

Similarly, we define

$$
\tilde{\mathscr{F}}_{i}^{a \eta}\left(x_{B}, z_{\perp}\right) \equiv \frac{2}{s} \int d z_{*} e^{-i x_{B} z_{*}} g\left(\tilde{F}_{\bullet i}^{m}\left(z_{*}, z_{\perp}\right)\left[z_{*}, \infty\right]_{z}^{m a}\right)^{\eta}
$$


in the complex-conjugate part of the amplitude.

In this notations the unintegrated gluon TMD (1.1) can be represented as

$$
\begin{aligned}
& \left\langle p\left|\tilde{\mathscr{F}}_{i}^{a \eta}\left(x_{B}, z_{\perp}\right) \mathscr{F}^{a i \eta}\left(x_{B}, 0_{\perp}\right)\right| p+\xi_{p_{2}}\right\rangle \equiv \sum_{X}\left\langle p\left|\tilde{\mathscr{F}}_{i}^{a \eta}\left(x_{B}, z_{\perp}\right)\right| X\right\rangle\left\langle X\left|\mathscr{F}^{a i \eta}\left(x_{B}, 0_{\perp}\right)\right| p+\xi_{p_{2}}\right\rangle \\
& =-4 \pi^{2} \delta(\xi) x_{B} g^{2} \mathscr{D}\left(x_{B}, z_{\perp}, \eta\right)
\end{aligned}
$$

Hereafter we use a short-hand notation

$$
\left\langle p\left|\tilde{\mathscr{O}}_{1} \ldots \tilde{\mathscr{O}}_{m} \mathscr{O}_{1} \ldots \mathscr{O}_{n}\right| p^{\prime}\right\rangle \equiv \sum_{X}\left\langle p\left|\tilde{T}\left\{\tilde{\mathscr{O}}_{1} \ldots \tilde{\mathscr{O}}_{m}\right\}\right| X\right\rangle\left\langle X\left|T\left\{\mathscr{O}_{1} \ldots \mathscr{O}_{n}\right\}\right| p^{\prime}\right\rangle
$$

where tilde on the operators in the 1.h.s. of this formula stands as a reminder that they should be inverse time ordered as indicated by inverse-time ordering $\tilde{T}$ in the r.h.s. of the above equation.

As discussed e.g. in Ref. [7], such martix element can be represented by a double functional integral

$$
\begin{aligned}
& \left\langle\tilde{\mathscr{O}}_{1} \ldots \tilde{\mathscr{O}}_{m} \mathscr{O}_{1} \ldots \mathscr{O}_{n}\right\rangle \\
& =\int D \tilde{A} D \tilde{\psi} D \tilde{\psi} e^{-i S_{\mathrm{QCD}}(\tilde{A}, \tilde{\psi})} \int D A D \bar{\psi} D \psi e^{i S_{\mathrm{QCD}}(A, \psi)} \tilde{\mathscr{O}}_{1} \ldots \tilde{\mathscr{O}}_{m} \mathscr{O}_{1} \ldots \mathscr{O}_{n}
\end{aligned}
$$

with the boundary condition $\tilde{A}(\vec{x}, t=\infty)=A(\vec{x}, t=\infty)$ (and similarly for quark fields) reflecting the sum over all intermediate states $X$. Due to this condition, the matrix element (2.3) can be made gauge-invariant by connecting the endpoints of Wilson lines at infinity with the gauge link

$$
\left\langle p\left|\tilde{\mathscr{F}}_{i}^{a}\left(x_{B}, x_{\perp}\right) \mathscr{F}^{a i}\left(x_{B}^{\prime}, y_{\perp}\right)\right| p^{\prime}\right\rangle \rightarrow\left\langle p\left|\tilde{\mathscr{F}}_{i}^{a}\left(x_{B}, x_{\perp}\right)\left[x_{\perp}+\infty p_{1}, y_{\perp}+\infty p_{1}\right] \mathscr{F}^{a i}\left(\beta_{B}^{\prime}, y_{\perp}\right)\right| p^{\prime}\right\rangle(2.6)
$$

This gauge link is important if we use the light-like gauge $p_{1}^{\mu} A_{\mu}=0$ for calculations but in all other gauges it can be neglected. We will not write it down explicitly but will always assume it in our formulas.

\subsection{Evolution kernel in the leading order}

We will study the rapidity evolution of the operator

$$
\tilde{\mathscr{F}}_{i}^{a \eta}\left(x_{B}, x_{\perp}\right) \mathscr{F}_{j}^{a \eta}\left(x_{B}, y_{\perp}\right)
$$

Our aim is to construct an evolution of the TMD operator with $\eta=\ln \sigma$, where $\sigma$ is a corresponding cutoff in $\alpha$. Tofindthe evolution kernel, we introduce a new cutoff $\eta^{\prime}$ and integrate over "slow" fields with rapidity $\eta^{\prime}<y<\eta$, where $y=\ln \alpha$. We suppose that the "fast" fields with $y<\eta^{\prime}$ are fixed and serve as a background field. The typical diagrams are given in Fig. 1.

We aim to obtain evolution at the one loop order so there is only one "quantum" (blue) line of the "slow" fields propagating in the "classical" (red) background of the "fast" fields. In calculation of this diagrams we distinguish between two limits: $k_{\perp}^{2} \sim l_{\perp}^{2}$ and $k_{\perp}^{2} \gg l_{\perp}^{2}$, where $k_{\perp}$ is a transverse momentum of the "quantum" fields, while $l_{\perp}$ is the characteristic transverse scale of the external fields.

It is easy to understand that the typical longitudinal distance traveled by the "slow" fields is $\alpha s / k_{\perp}^{2} \sim \sigma s / k_{\perp}^{2}$. At the same time the longitudinal scale of the "fast" fields is $\sigma_{*} \sim \sigma^{\prime} s / l_{\perp}^{2}$. We 


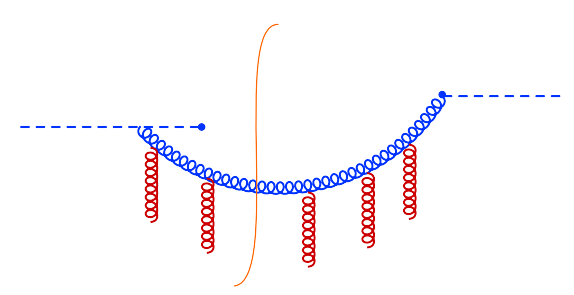

(a)

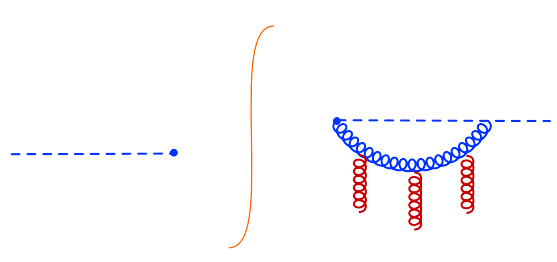

(b)

Figure 1: Typical diagrams for production (a) and virtual (b) contributions to the evolution kernel. The dashed lines denote gauge links.

suppose that $\sigma \gg \sigma^{\prime}$, so in the limit $k_{\perp}^{2} \sim l_{\perp}^{2}$ the "quantum" fields effectively propagate through a thin area of the "fast" fields of the background. This is the shock-wave approximation: the red (external) fields in Fig. 1 are concentrated within a segment $\left(-\sigma_{*}, \sigma\right)$. Of course this segment is very short, but in our calculation it is nevertheless finite. We consider propagation of the "quantum" (blue) fields through this segment and expand the corresponding propagator in powers of $\frac{k_{\perp}^{2}}{\alpha s} \sigma * \sim$ $\frac{\sigma^{\prime}}{\sigma} \ll 1$.

However, in the limit $k_{\perp} \gg l_{\perp}$ this scheme is not valid: there is no separation in distance between the "slow" and "fast" fields. Insteed of the shock-wave approximation, in this case we apply the light-cone expansion approach [8]. We take a ration $l_{\perp} / k_{\perp} \ll 1$ as an expansion parameter and expand the gluon propagator in Fig. 1 around the light ray $y_{\perp}+\frac{2}{s} y_{*} p_{1}$, which is the direction of the Wilson line in definition (1.1).

\subsection{The Lipatov vertex}

As usual, there are two contributions into the evolution kernel: the real gluon emission (Fig. 1a) and the virtual diagrams (Fig. 1b). The "real" correction to the TMD operator (2.7) is

$$
\left\langle\tilde{\mathscr{F}}_{i}^{a}\left(x_{\perp}, x_{B}\right) \mathscr{F}_{j}^{a}\left(y_{\perp}, x_{B}\right)\right\rangle^{\ln \sigma}=-\int_{\sigma^{\prime}}^{\sigma} \frac{d \alpha}{2 \alpha} d^{2} k_{\perp}\left(\tilde{L}_{i}^{b a ; \mu}\left(k, x_{\perp}, x_{B}\right) L_{\mu j}^{a b}\left(k, y_{\perp}, x_{B}\right)\right)^{\ln \sigma^{\prime}},
$$

where the Lipatov vertex $L_{\mu i}^{a b}\left(k, y_{\perp}, x_{B}\right)=i \lim _{k^{2} \rightarrow 0} k^{2}\left\langle A_{\mu}^{a}(k) \mathscr{F}_{i}^{b}\left(y_{\perp}, x_{B}\right)\right\rangle$ is an amplitude of the single-gluon production by the operator $\mathscr{F}_{i}^{b}$. There are several possible types of emission. The corresponding diagrams can be found in Fig. 2. We start with the calculation of the Lipatov vertex

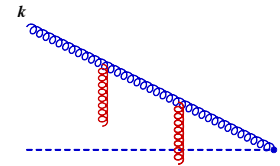

(a)

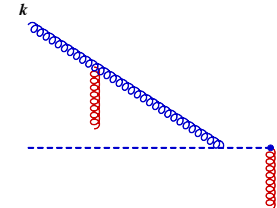

(b)

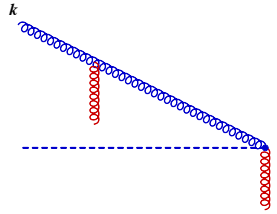

(c)

Figure 2: Diagrams contributing to Lipatov vertex

in the light-cone approach. We expand the gluon propagator around direction of the Wilson line and neglect terms suppressed by $l_{\perp} / k_{\perp} \ll 1$. One can show that the leading contribution comes from 
the terms linear in the gluon strength tensor $F_{\bullet}$. The external (red) field has no specific longitudinal properties and we take into account all possible emissions from the Wilson line and the strength tensor in the definition (1.2) as well. The details of the calculation can be found in [9]. Here we show only final result:

$$
\begin{aligned}
& L_{\mu i}^{a b}\left(k, y_{\perp}, x_{B}\right)=i \lim _{k^{2} \rightarrow 0} k^{2}\left\langle A_{\mu}^{a \mathrm{q}}(k)\left(\mathscr{F}_{i}^{b}\left(x_{B}, y_{\perp}\right)\right)^{1 \mathrm{st}}\right\rangle=\frac{2 g e^{-i(k, y)}}{\alpha x_{B} s+k_{\perp}^{2}} \\
& \times\left[\frac{\alpha x_{B} s}{k_{\perp}^{2}}\left(\frac{k_{\perp}^{2}}{\alpha s} p_{2 \mu}-\alpha p_{1 \mu}\right) \delta_{i}^{l}-\delta_{\mu}^{l} k_{i}+\frac{\alpha x_{B} s g_{\mu i} k^{l}}{k_{\perp}^{2}+\alpha x_{B} s}+\frac{2 \alpha k_{i} k^{l}}{k_{\perp}^{2}+\alpha x_{B} s} p_{1 \mu}\right] \mathscr{F}_{l}^{a b}\left(x_{B}+\frac{k_{\perp}^{2}}{\alpha s}, y_{\perp}\right) .
\end{aligned}
$$

On the other hand, in the limit $k_{\perp} \sim l_{\perp}$ we apply the shock-wave approximation. In this case the external field in Fig. 2 shrinks to fill a small area from $-\sigma_{*}$ to $\sigma_{*}$ in longitudinal direction. Effectively the diagrams in Fig. 2 are the diagrams in the shock-wave background, see Fig. 3. Now

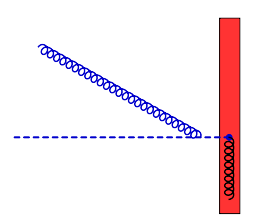

(a)

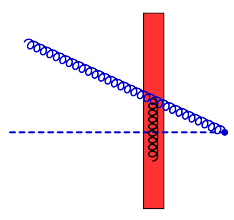

(b)

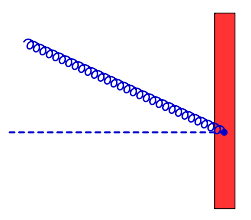

(c)

Figure 3: Lipatov vertex in the shock-wave approximation

we take into account not only different types of emission, but the position of the emission point as well: we separate the emission from the left, right sides and from inside of the shock-wave. The sum of all diagrams in Fig. 3 is

$$
\begin{aligned}
& L_{\mu i}^{a b}\left(k, y_{\perp}, x_{B}\right)=2 g e^{-i(k, y) \perp}\left(\frac{p_{2 \mu}}{\alpha s}-\frac{\alpha p_{1 \mu}}{k_{\perp}^{2}}\right)\left[\mathscr{F}_{i}\left(x_{B}, y_{\perp}\right)-U_{i}\left(y_{\perp}\right)\right]^{a b} \\
& +g\left(k_{\perp} \mid g_{\mu i}\left(\frac{\alpha x_{B} s}{\alpha x_{B} s+p_{\perp}^{2}}-U \frac{\alpha x_{B} s}{\alpha x_{B} s+p_{\perp}^{2}} U^{\dagger}\right)+2 \alpha p_{1 \mu}\left(\frac{p_{i}}{\alpha x_{B} s+p_{\perp}^{2}}-U \frac{p_{i}}{\alpha x_{B} s+p_{\perp}^{2}} U^{\dagger}\right)\right. \\
& \left.+\left[2 i x_{B} p_{2 \mu} \partial_{i} U-2 i \partial_{\mu}^{\perp} U p_{i}+\frac{2 p_{2 \mu}}{\alpha s} \partial_{\perp}^{2} U p_{i}\right] \frac{1}{\alpha x_{B} s+p_{\perp}^{2}} U^{\dagger}-\frac{2 \alpha p_{1 \mu}}{p_{\perp}^{2}} U_{i} \mid y_{\perp}\right)^{a b} .
\end{aligned}
$$

In this calculation we expand the "slow" gluon propagator in powers of the size of the shock-wave and neglect terms suppressed by $\frac{p_{\perp}^{2}}{\alpha s} \sigma_{*} \ll 1$.

Next, we combine the two results (2.9 and (2.10) in an interpolation formula:

$$
\begin{aligned}
& L_{\mu i}^{a b}\left(k, y_{\perp}, x_{B}\right) \\
& =g\left(k_{\perp} \mid \frac{\alpha x_{B} s g_{\mu i}+2 \alpha p_{1 \mu} k_{i}}{\alpha x_{B} s+k_{\perp}^{2}}\left(2 i k^{j} \partial_{j} U-\partial_{\perp}^{2} U\right) \frac{1}{\alpha x_{B} s+p_{\perp}^{2}} U^{\dagger}+2 i \alpha p_{1 \mu} \partial_{i} U \frac{1}{\alpha x_{B} s+p_{\perp}^{2}} U^{\dagger}\right. \\
& \left.+\frac{2 i}{\alpha s} p_{2 \mu} \partial_{i} U \frac{\alpha x_{B} s}{\alpha x_{B} s+p_{\perp}^{2}} U^{\dagger}-\left[2 i \partial_{\mu} U-\frac{2 p_{2 \mu}}{\alpha s} \partial_{\perp}^{2} U\right] \frac{p_{i}}{\alpha x_{B} s+p_{\perp}^{2}} U^{\dagger}-\frac{2 \alpha p_{1 \mu}}{p_{\perp}^{2}} i\left(\partial_{i} U\right) U^{\dagger} \mid y_{\perp}\right)^{a b} \\
& +\frac{2 g e^{-i(k, y)}}{\alpha x_{B} s+k_{\perp}^{2}}\left[-\delta_{\mu}^{j} k_{i}+\frac{2 \alpha k_{i} k^{j} p_{1 \mu}}{\alpha x_{B} s+k_{\perp}^{2}}+\frac{\alpha x_{B} s g_{\mu i} k^{j}}{\alpha x_{B} s+k_{\perp}^{2}}+x_{B} p_{2 \mu} \delta_{i}^{j}-\alpha p_{1 \mu} \frac{\alpha x_{B} s}{k_{\perp}^{2}} \delta_{i}^{j}\right] \\
& \times\left[\mathscr{F}_{j}\left(x_{B}+\frac{k_{\perp}^{2}}{\alpha s}, y_{\perp}\right)-U_{j}\left(y_{\perp}\right)\right]^{a b} .
\end{aligned}
$$


In Ref. [9] we prove that it is correct in the whole region of values of $x_{B}$. It is also important that (2.11) doesn't break the gauge invariance: $k^{\mu} L_{\mu i}^{a b}\left(k, y_{\perp}, x_{B}\right)=0$. Finally, we use the Lipatov vertex (2.11) to calculate the "real" part of the evolution kernel according to (2.8).

\subsection{The virtual emission}

The second part of the evolution kernel is the virtual correction, see Fig. 1b. For calculation of this diagrams we apply the same strategy we used in derivation of the Lipatov vertex. In the limit $k_{\perp} \gg l_{\perp}$ we expand the gluon propagator around $y_{\perp}+\frac{2}{s} y_{*} p_{1}$ direction and neglect terms suppressed by $l_{\perp} / k_{\perp} \ll 1$. What is left is used in the explicit calculation of the diagrams if Fig. 1b. (There are actually several of them, see [9]). In the limit $k_{\perp} \sim l_{\perp}$ we use the shock-wave approximation so the

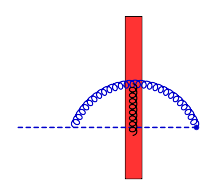

(a)

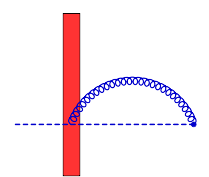

(b)

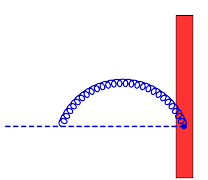

(c)

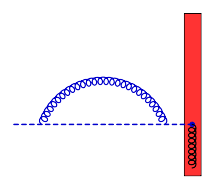

(d)

Figure 4: The virtual correction in the shock-wave approximation

virtual emission diagrams take a form given in Fig. 4. We show that the sum of this diagrams can be combined with the result obtained at $k_{\perp} \gg l_{\perp}$ and one can write an expression which describes both limits and is valid for all values of $x_{B}$ :

$$
\begin{aligned}
& \left\langle\mathscr{F}_{i}^{n}\left(y_{\perp}, x_{B}\right)\right\rangle^{\mathrm{virt}}= \\
& =-i g^{2} f^{n k l} \int_{\sigma^{\prime}}^{\sigma} \frac{d \alpha}{\alpha}\left(y_{\perp}\left|\frac{p^{j}}{p_{\perp}^{2}}\left(2 \partial_{i} \partial_{j} U+g_{i j} \partial_{\perp}^{2} U\right) \frac{1}{\alpha x_{B} s+p_{\perp}^{2}} U^{\dagger}+\frac{\alpha x_{B} s p_{\perp}^{-2}}{\alpha x_{B} s+p_{\perp}^{2}} \mathscr{F}_{i}\left(x_{B}\right)\right| y_{\perp}\right)^{k l} .
\end{aligned}
$$

\section{Evolution equation for gluon TMD}

Now we can write the one-loop correction to the operator (1.1). It is a sum of the product of two Lipatov vertexes (2.11) and the virtual contribution (2.12):

$$
\begin{aligned}
& \left\langle\tilde{\mathscr{F}}_{i}^{a}\left(x_{\perp}, x_{B}\right) \mathscr{F}_{j}^{a}\left(y_{\perp}, x_{B}\right)\right\rangle_{\text {one-loop }}^{\ln \sigma}=-\int_{\sigma^{\prime}}^{\sigma} \frac{d \alpha}{2 \alpha} d^{2} k_{\perp}\left(\tilde{L}_{i}^{b a ; \mu}\left(k, x_{\perp}, x_{B}\right) L_{\mu j}^{a b}\left(k, y_{\perp}, x_{B}\right)\right)^{\ln \sigma^{\prime}} \\
& +\tilde{\mathscr{F}}_{i}^{a}\left(x_{\perp}, x_{B}\right)\left\langle\mathscr{F}_{j}^{a}\left(y_{\perp}, x_{B}\right)\right\rangle^{\text {virt }}+\left\langle\tilde{\mathscr{F}}_{i}^{a}\left(x_{\perp}, x_{B}\right)\right\rangle^{\text {virt } \mathscr{F}_{j}^{a}\left(y_{\perp}, x_{B}\right) .}
\end{aligned}
$$

To get the evolution equation we differentiate this expression over $\sigma$. Combining all results together we get

$$
\begin{aligned}
& \frac{d}{d \log \sigma}\left\langle p\left|\tilde{\mathscr{F}}_{i}^{a}\left(x_{\perp}, x_{B}\right) \mathscr{F}_{j}^{a}\left(y_{\perp}, x_{B}\right)\right| p\right\rangle \\
& =-\alpha_{s}\langle p| \operatorname{Tr}\left\{\int d ^ { 2 } k _ { \perp } \theta ( 1 - x _ { B } - \frac { k _ { \perp } ^ { 2 } } { \sigma s } ) \left[\left(x_{\perp} \mid\left(\tilde{U} \frac{1}{\sigma x_{B} s+p_{\perp}^{2}}\left(\tilde{U}^{\dagger} k_{k}+p_{k} \tilde{U}^{\dagger}\right)\right.\right.\right.\right. \\
& \left.\left.\times \frac{\sigma x_{B} s g_{\mu i}-2 k_{\mu}^{\perp} k_{i}}{\sigma x_{B} s+k_{\perp}^{2}}-2 k_{\mu}^{\perp} g_{i k} \tilde{U} \frac{1}{\sigma x_{B} s+p_{\perp}^{2}} \tilde{U}^{\dagger}-2 g_{\mu k} \tilde{U} \frac{p_{i}}{\sigma x_{B} s+p_{\perp}^{2}} \tilde{U}^{\dagger}\right) \tilde{\mathscr{F}}^{k}\left(x_{B}+\frac{k_{\perp}^{2}}{\sigma s}\right) \mid k_{\perp}\right)
\end{aligned}
$$




$$
\begin{aligned}
& \times\left(k_{\perp} \mid \mathscr{F}^{l}\left(x_{B}+\frac{k_{\perp}^{2}}{\sigma s}\right)\left(\frac{\sigma x_{B} s \delta_{j}^{\mu}-2 k_{\perp}^{\mu} k_{j}}{\sigma x_{B} s+k_{\perp}^{2}}\left(k_{l} U+U p_{l}\right) \frac{1}{\sigma x_{B} s+p_{\perp}^{2}} U^{\dagger}\right.\right. \\
& \left.\left.-2 k_{\perp}^{\mu} g_{j l} U \frac{1}{\sigma x_{B} s+p_{\perp}^{2}} U^{\dagger}-2 \delta_{l}^{\mu} U \frac{p_{j}}{\sigma x_{B} s+p_{\perp}^{2}} U^{\dagger}\right) \mid y_{\perp}\right) \\
& +2\left(x_{\perp}\left|\tilde{\mathscr{F}}_{i}\left(x_{B}+\frac{k_{\perp}^{2}}{\sigma s}\right)\right| k_{\perp}\right)\left(k_{\perp} \mid \mathscr{F}^{l}\left(x_{B}+\frac{k_{\perp}^{2}}{\sigma s}\right)\left(\frac{k_{j}}{k_{\perp}^{2}} \frac{\sigma x_{B} s+2 k_{\perp}^{2}}{\sigma x_{B} s+k_{\perp}^{2}}\left(k_{l} U+U p_{l}\right) \frac{1}{\sigma x_{B} s+p_{\perp}^{2}} U^{\dagger}\right.\right. \\
& \left.\left.+2 U \frac{g_{j l}}{\sigma x_{B} s+p_{\perp}^{2}} U^{\dagger}-2 \frac{k_{l}}{k_{\perp}^{2}} U \frac{p_{j}}{\sigma x_{B} s+p_{\perp}^{2}} U^{\dagger}\right) \mid y_{\perp}\right) \\
& +2\left(x_{\perp} \mid\left(\tilde{U} \frac{1}{\sigma x_{B} s+p_{\perp}^{2}}\left(\tilde{U}^{\dagger} k_{k}+p_{k} \tilde{U}^{\dagger}\right) \frac{k_{i}}{k_{\perp}^{2}} \frac{\sigma x_{B} s+2 k_{\perp}^{2}}{\sigma x_{B} s+k_{\perp}^{2}}+2 \tilde{U} \frac{g_{i k}}{\sigma x_{B} s+p_{\perp}^{2}} \tilde{U}^{\dagger}\right.\right. \\
& \left.\left.\left.-2 \tilde{U} \frac{p_{i}}{\sigma x_{B} s+p_{\perp}^{2}} \tilde{U}^{\dagger} \frac{k_{k}}{k_{\perp}^{2}}\right) \tilde{\mathscr{F}}^{k}\left(x_{B}+\frac{k_{\perp}^{2}}{\sigma s}\right) \mid k_{\perp}\right)\left(k_{\perp}\left|\mathscr{F}_{j}\left(x_{B}+\frac{k_{\perp}^{2}}{\sigma s}\right)\right| y_{\perp}\right)\right] \\
& +2 \tilde{\mathscr{F}}_{i}\left(x_{\perp}, x_{B}\right)\left(y_{\perp}\left|-\frac{p^{m}}{p_{\perp}^{2}} \mathscr{F}_{k}\left(x_{B}\right)\left(i \overleftarrow{\partial}_{l}+U_{l}\right)\left(2 \delta_{m}^{k} \delta_{j}^{l}-g_{j m} g^{k l}\right) U \frac{1}{\sigma x_{B} s+p_{\perp}^{2}} U^{\dagger}\right| y_{\perp}\right) \\
& +2\left(x_{\perp}\left|\tilde{U} \frac{1}{\sigma x_{B} s+p_{\perp}^{2}} \tilde{U}^{\dagger}\left(2 \delta_{i}^{k} \delta_{m}^{l}-g_{i m} g^{k l}\right)\left(i \partial_{k}-\tilde{U}_{k}\right) \tilde{\mathscr{F}}_{l}\left(x_{B}\right) \frac{p^{m}}{p_{\perp}^{2}}\right| x_{\perp}\right) \mathscr{F}_{j}\left(y_{\perp}, x_{B}\right) \\
& -4 \int \frac{d^{2} k_{\perp}}{k_{\perp}^{2}}\left[\theta\left(1-x_{B}-\frac{k_{\perp}^{2}}{\sigma s}\right) \tilde{\mathscr{F}}_{i}\left(x_{\perp}, x_{B}+\frac{k_{\perp}^{2}}{\sigma s}\right) \mathscr{F}_{j}\left(y_{\perp}, x_{B}+\frac{k_{\perp}^{2}}{\sigma s}\right) e^{i(k, x-y) \perp}\right. \\
& \left.\left.-\frac{\sigma x_{B} s}{\sigma x_{B} s+k_{\perp}^{2}} \tilde{\mathscr{F}_{i}}\left(x_{\perp}, x_{B}\right) \mathscr{F}_{j}\left(y_{\perp}, x_{B}\right)\right]\right\}|p\rangle+O\left(\alpha_{s}^{2}\right) .
\end{aligned}
$$

The kinematical constraint $k_{\perp}^{2} \leq \alpha\left(1-\beta_{B}\right) s$ in the production part of the amplitude reflects the fact that so the sum of the fraction $\beta_{B} p_{2}$ and the fraction $\frac{k_{\perp}^{2}}{\alpha s} p_{2}$ carried by the emitted gluon should be smaller than the initial hadron's momentum is $p \simeq p_{2}$.

The formula (3.2) is the main result of our study. It is an evolution equation of the gluon TMD operator (1.1) and is valid for all (except too small) values of transverse momentum $k_{\perp} \sim$ $\left(x_{\perp}-y_{\perp}\right)^{-1}$ and longitudinal momentum fraction variable $x_{B}$.

Let us discuss the gauge invariance of this equation. The 1.h.s. is gauge invariant after taking into account gauge link at $+\infty$ as shown in Eq. (2.6). As to the right side, it was obtained by calculation in the background field and promoting the background fields to operators in a usual way. However, we performed our calculations in a specific background field $A_{\bullet}\left(x_{*}, x_{\perp}\right)$ with a finite support in $x_{\perp}$ and we need to address the question how can we restore the r.h.s. of Eq. (3.2) in a generic field $A_{\mu}$. It is easy to see how one can restore the gauge-invariant form: just add gauge link at $+\infty p_{1}$ or $-\infty p_{1}$ appropriately. For example, the terms $U_{z}\left(z\left|\frac{1}{\sigma \beta s+p_{\perp}^{2}}\right| z^{\prime}\right) U_{z^{\prime}}^{\dagger}$ in r.h.s. of should be replaced by $U_{z}\left[z_{\perp}-\infty p_{1}, z_{\perp}^{\prime}-\infty p_{1}\right]\left(z\left|\frac{1}{\sigma \beta s+p^{2}}\right| z^{\prime}\right) U_{z^{\prime}}^{\dagger}$. After performing these insertions we will have the result which is (i) gauge invariant and (ii) coincides with Eq. (3.2) for our choice of background field. At this step, the background fields in the r.h.s. of Eq. (3.2) can be promoted to operators. However, the explicit display of these gauge links at $\pm \infty$ will make the evolution equation much less readable so we assume they are always in place rather than written explicitly.

It is demonstrated in Ref. [9] that the equation (3.2) has three correct limits: at $x \sim 1$ and large transverse momentum it yields the linear DGLAP equation, at $x \sim 1$ and small $k_{\perp}$ it gives the Sudakov double logarithm evolution, and at small $x$ it reduces to the non-linear equation of the 
Balitsky-Kovchegov (BK) type.

\section{Conclusions}

I have described the rapidity evolution of gluon TMD (1.1) in the whole range of Bjorken $x_{B}$ and the whole range of transverse momentum $k_{\perp}$. It should be emphasized that with our definition of rapidity cutoff (2.1) the leading-order matrix elements of TMD operators are UV-finite so the rapidity evolution is the only evolution and it describes all the dynamics of gluon TMDs (1.1) in the leading-log approximation.

As an outlook, it would be very interesting to obtain the NLO correction to the evolution equation (3.2). The NLO corrections to the BFKL and BK equation are available but they suffer from the well-known problem that they lead to negative cross sections. This difficulty can be overcome by the "collinear resummation" of double-logarithmic contributions for the BFKL [10] and BK [11] equations and we hope that our Eq. (3.2) and especially its future NLO version will help to solve the problem of negative cross sections of NLO amplitudes at high energies.

This work on TMD evolution was done in collaboration with A. Tarasov. In addition, the author is grateful G.A. Chirilli and A.V. Radyushkin for valuable discussions. The work was supported by contract DE-AC05-06OR23177 under which the Jefferson Science Associates, LLC operates the Thomas Jefferson National Accelerator Facility, and by the grant DE-FG02-97ER41028.

\section{References}

[1] J. C. Collins and D. E. Soper, Nucl. Phys. B194, 445 (1982).

[2] X. Ji, Jian-Ping Ma, and F. Yuan, Phys. Rev. D71, 034005 (2005).

[3] J.C. Collins, Foundations of Perturbative QCD (Cambridge University Press, Cambridge, 2011)

[4] P. J. Mulders and J. Rodrigues, Phys. Rev. D63, 094021 (2001).

[5] M.G. Echevarria, A. Idilbi, and I. Scimeni, JHEP 07002 (2012).

[6] J.C. Collins, D. E. Soper and G. Sterman, Nucl. Phys. B250, 199 (1985).

[7] I. Balitsky and V.M. Braun, Phys. Lett.B222, 121 (1989); Nucl. Phys. B361, 93 (1991); Nucl. Phys. B380, 51 (1992).

[8] I. Balitsky, Phys. Lett. B124, 230 (1983); I. Balitsky and V.M. Braun, Nucl. Phys. B311, 541 (1989).

[9] I. Balitsky and A. Tarasov, JHEP. 1510, 017 (2015)

[10] G.P. Salam, JHEP9807 019 (1998); M. Ciafaloni, D. Colferai, G.P. Salam, Phys.Rev. D60 114036 (1999); M. Ciafaloni, D. Colferai, G.P. Salam, and A.M. Stasto, Phys.Rev. D68 114003 (2003);

[11] L. Motyka and A. M. Stasto Phys.Rev. D79 085016 (2009); G. Beuf, Phys.Rev. D89 074039 (2014); E. Iancu, J.D. Madrigal, A.H. Mueller, G. Soyez, D.N. Triantafyllopoulos, Phys.Lett. B744 293 (2015). 